Document downloaded from:

http://hdl.handle.net/10251/120674

This paper must be cited as:

Cogollos, S.; Vague Cardona, JJ.; Boria Esbert, VE.; Martínez Pérez, JD. (2018). Novel Planar and Waveguide Implementations of Impedance Matching Networks Based on Tapered Lines Using Generalized Superellipses. IEEE Transactions on Microwave Theory and Techniques. 66(4):1874-1884. https://doi.org/10.1109/TMTT.2018.2791952

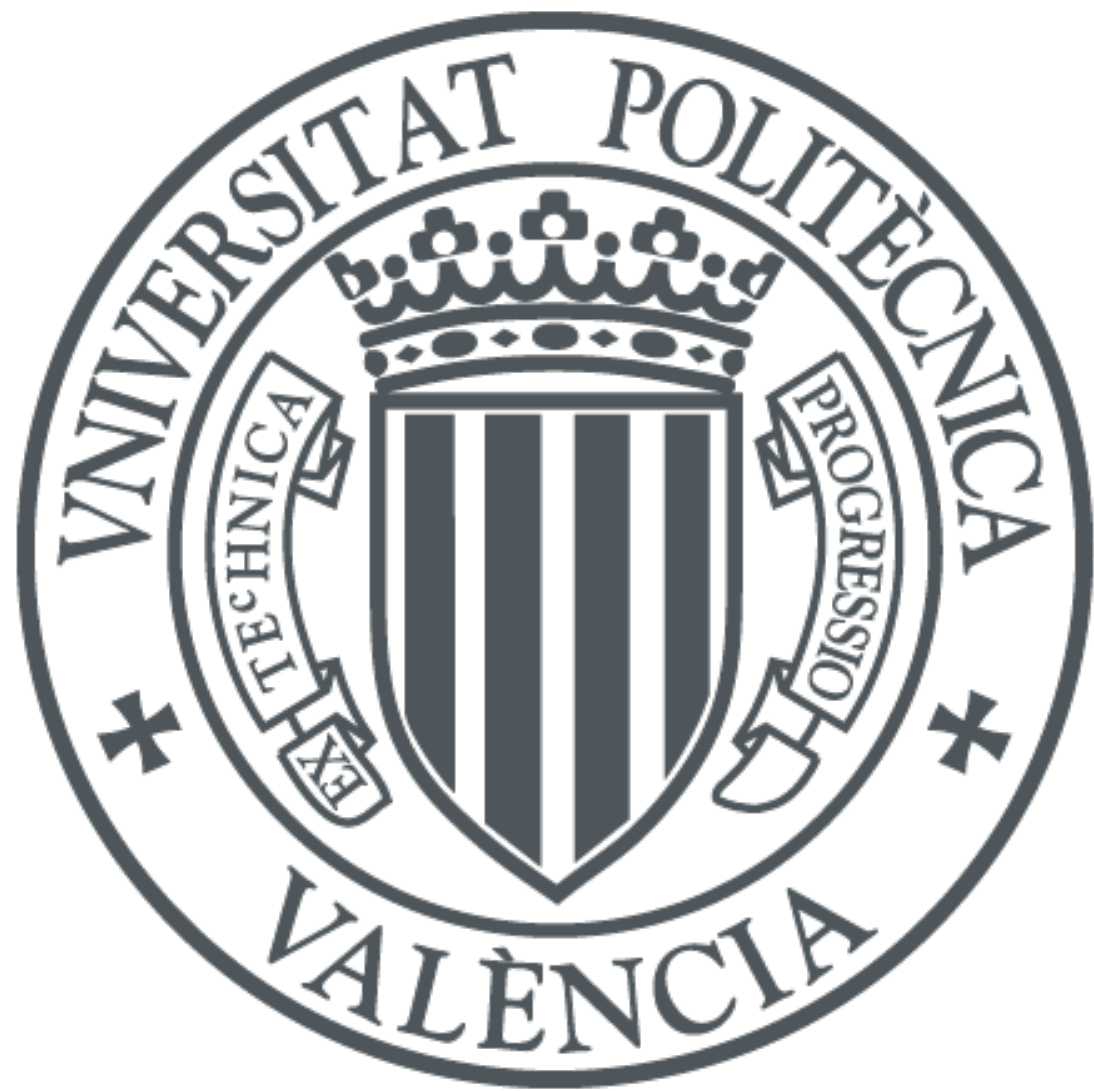

The final publication is available at

http://doi.org/10.1109/TMTT.2018.2791952

Copyright Institute of Electrical and Electronics Engineers

Additional Information

(c) 2018 IEEE. Personal use of this material is permitted. Permission from IEEE must be obtained for all other users, including reprinting/ republishing this 


\title{
Novel Planar and Waveguide Implementations of Impedance Matching Networks Based on Tapered Lines Using Generalized Superellipses
}

\author{
Santiago Cogollos, Member, IEEE, Joaquin Vague, Vicente E. Boria Member, IEEE, \\ Jorge D. Martínez Member, IEEE,
}

\begin{abstract}
For the practical implementation of RF and microwave impedance matching networks, a widely employed solution -alternative to the use of classical impedance transformers- is based on tapered lines. This paper shows a simple method to design smooth tapers, that take into account the dispersion of the line and the required design bandwidth simultaneously. A planar taper has been designed in microstrip technology with the same length of classical ones but improving their performances. A waveguide prototype has been also designed with similar performance to a commercial one but with one third of its length. Both tapered structures have been obtained through optimization of very few parameters using the same design strategy. As a result, the reflection coefficient of the tapers can be optimally adapted to a given specific mask using the prescribed value of physical length. Experimental results for both tapers are included for validation of the proposed topologies and the related design method.
\end{abstract}

Index Terms-Impedance matching, nonuniform transmission lines, microwave circuits, planar circuits, waveguides.

\section{INTRODUCTION}

I MPEDANCE MATCHING is an old problem in RF and microwave technology, arising when two sections of different transmission lines have to be connected properly (i.e. minimizing potential return losses due to the mismatch of associated characteristic impedances). The first approach and the simplest one is the single- or multi-section transformer consisting of quarter-wavelength sections of transmission lines [1]. Here, the goal is usually an equirriple reflection coefficient over a certain bandwidth, which is directly related to the considered number of sections. However, soon appeared some reasons to avoid this solution. As a first reason, the higher the bandwidth the higher the number of required matching sections, therefore the total length is the number of sections multiplied by $\lambda_{g} / 4$ (being $\lambda_{g}$ the wavelength on each transmission line section). These methods do not allow to fix a certain length as a design parameter. Another reason to avoid the multi-section transformer is the inconvenience of the impedance steps. Adding edges, corners or abrupt changes inside the structure, avoiding a smooth profile of the involved transmission line or waveguide, is a challenge in the manufacturing process at high frequencies. Additionally, the impedance steps introduce reactances which may be

S. Cogollos, J. Vague, V.E. Boria and J. D. Martínez are with Universitat Politècnica de València, Camino de Vera $\mathrm{s} / \mathrm{n}, 46022$, Valencia, Spain. Email: sancobo@dcom.upv.es, jvague@dcom.upv.es, vboria@dcom.upv.es, jdmartinez@eln.upv.es.. This work has been partially supported by MINECO (Spanish Government) under R\&D project TEC2016-75934-C4-1R. Manuscript submitted June 29, 2017, revised September 26, 2017.
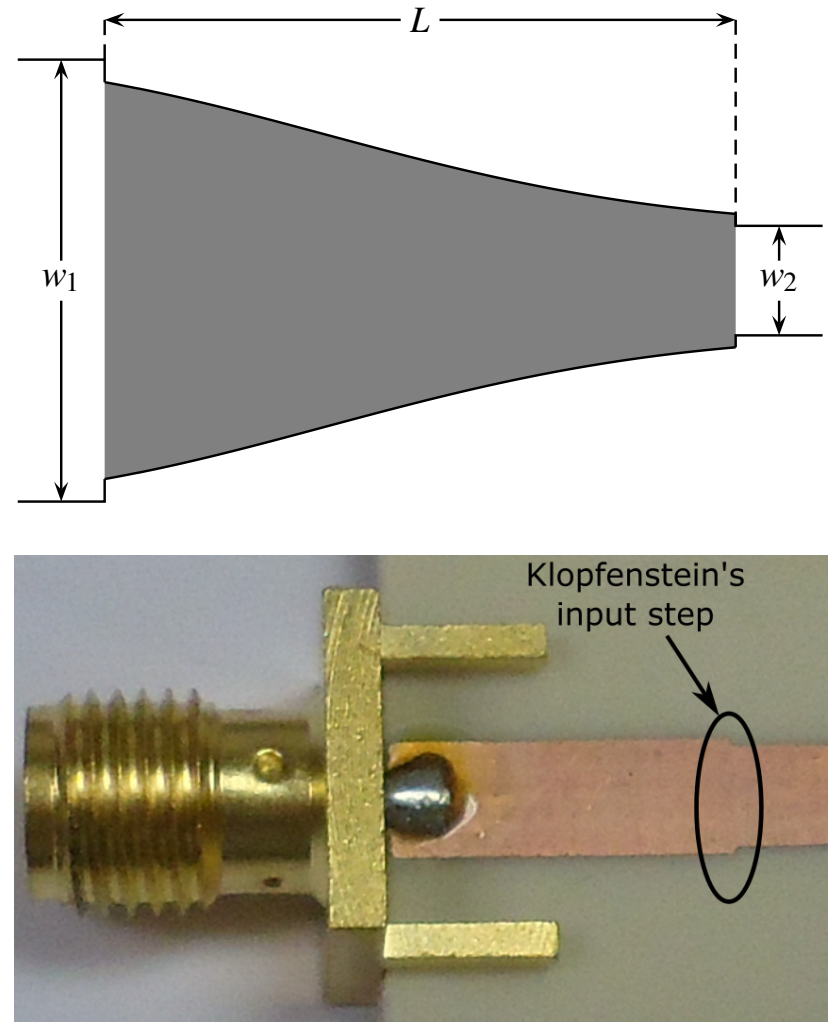

Fig. 1. Top: Klopfenstein (Dolph-Chebyshev) taper of length $L$ used to match lines with different widths $w_{1}$ and $w_{2}$. The steps at the input and output, inherent to this taper, are clearly shown. Bottom: Detail of a prototype of a Klopfenstein taper where the input step is shown.

compensated, but complicating the design. Moreover, these sharp corners produce the excitation of higher order modes hard to predict at the design stage.

In the quest for the optimal solution, tapered lines were designed using simple curve approximations only for planar designs. Linear, triangular and exponential tapers provided a simple profile for a given length [2], [3]. The clear advantage of a typical taper is the matching, not in a given bandwidth but from a certain minimum frequency $f_{\text {min }}$.

Finally, the Klopfenstein (Dolph-Chebyshev) taper appeared giving the optimal solution (in terms of equirriple response) for planar realizations [4]. The same solution, but using a different approach was obtained in the same year [5], pointing to a slight flaw in Klopfenstein's solution that was definitely corrected in [6]. However, this optimal solution shows certain limitations and drawbacks. The first drawback is the lack of 
smoothness because there are two steps (at the input and at the output) inherent to this design (see top of Fig. 1). When this ideal design is built, the steps cannot be easily manufactured as shown at the bottom of Fig. 1. Steps are not desired if excitation of spurious modes must be avoided. The second issue is the final design formula in terms of impedances. This is very convenient if the relationship impedance-frequency is constant. However, one of the main assumptions in [5] is that the characteristic impedance is fixed with frequency, and the impedance formula is given for $f_{\min }$. These facts can be tolerable for microstrip designs [7], but unacceptable for waveguide structures where characteristic impedance depends on the height-width ratio $b / a$ (see [5] and [8]).

In [9], the degradation at high frequencies was handled, focusing on the fact that, in practice, an infinite bandwidth is never needed. Therefore, from certain frequency upwards, the degradation is unimportant. Using this line of thought, a shorter taper than the optimum one can be designed for the same performance within a required bandwidth.

In the line of avoiding inconveniences due to the steps of the Klopfenstein's taper, near-optimum designs were developed. A smooth solution was given in [10], whose only drawback was related to the loss of the equirriple behavior.

Another remarkable work, based on a solution in series form similar to [5] but with a controllable equirriple response, was given in [11]. Its final aim was to place the reflection zeros in desired locations to control the ripple according to the designers' criteria.

However, theoretical designs like [4], [5], [10] and [11] were developed with some assumptions: TEM mode propagation and characteristic impedance constant with frequency. Empirically, it is well-known that this fact is not seen in practice. Here, the dispersion starts to play an important role in several aspects:

- Spatial dispersion: permittivity $\epsilon$ or conductivity $\sigma$ have dependence on the wavenumber [12] (in guided signals $\beta$ takes the role of the wavenumber $k$ ). In general, any guided signal varies with the term $e^{j(\omega t-\beta z)}$. If $\epsilon=\epsilon(\beta)$ and $\sigma=\sigma(\beta)$ this phenomenon can be an issue.

- Frequency dispersion: phase velocity have dependence on the signal frequency. It can be either monomode or multimode.

Controlling the value of the permittivity of the material along the length of the taper, a matching is also possible even with classical techniques [5]. It is remarkable that new advances controlling the dispersion (mainly in planar technologies) based on metamaterials are still in the early stages. These structures are based on cells with quasi-periodic arrangements, where losses may be still high enough, but promising results are recently shown in [13], [14].

In general, the main objective of this work is to consider in a simple manner any type of dispersion and practical issues that can be included in a EM simulator. For instance, it is widely known that the characteristic impedance of a microstrip line is frequency dependent, and shows a positive increase with an increase in frequency [15]. Inclusion of dispersion starts to be important in microwave designs [16], with the main goal of

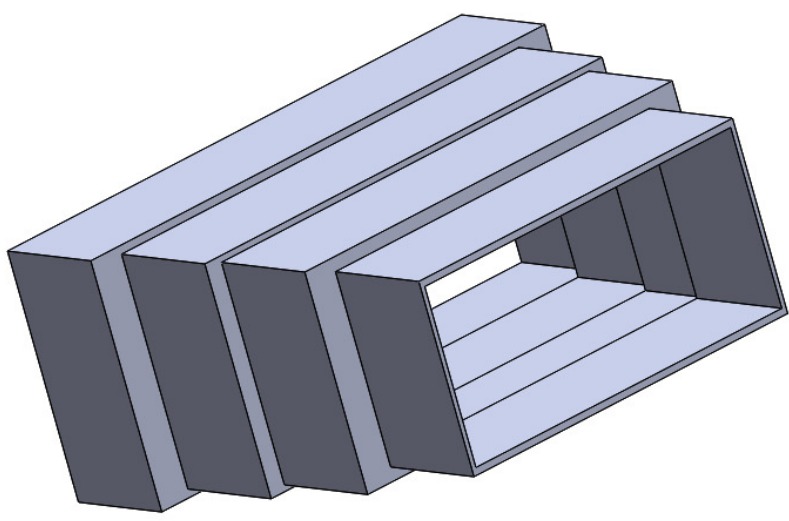

Fig. 2. 3D-view schematic of a classical four-section transformer in waveguide technology.

approximating the real behavior and EM simulations as much as possible.

Further advances have been carried out in this field. In the synthesis framework, [17] included loss and dispersion, but the response is computed solving the approximate Ricatti's equation instead of using EM solvers. Additionally, specific tapers have been analyzed by solving numerically the Ricatti's equation including realistic modelling (e.g. fin-line tapers [18]). Very recently, an efficient method has been developed to analyze tapers using circuit models at the expense of complicating the related theory [19].

Another strong motivation of our work was the potential implementation of taper solutions in [4], [5], [10] and [11] with waveguide technology, which is not easy since the characteristic impedance in such technology involves one additional degree of freedom (when compared with planar technologies). The width $a$ and the height $b$ of the waveguide simultaneously affect the waveguide characteristic impedance [20], and although the previous methods are extensible in terms of impedances, there is no clue about the best relationship between $a$ and $b$ to achieve both a smooth profile and the best performance in terms of frequency at the same time.

Therefore, the aim of this paper is to provide an alternative smooth taper profile (in both planar and waveguide technologies) accounting for the dispersion, the bandwidth and a flexible reflection requirement (not necessarily equirriple) using a simple optimization algorithm. The algorithm provides the parameters (usually two for planar and four for waveguide designs) of a generalized superelliptic curve that will be the profile of the proposed novel taper.

This new design method can be applied not only to planar topologies, as it was successfully shown in [21], but also to waveguide technology where modal dispersion is an issue. In fact, it is for waveguide technology where this method provides a higher performance, using the same design procedure with only subtle changes in its implementation that are fully described in this paper.

Traditionally, waveguide multi-section transformers (see Fig. 2) are designed using several methods. Among them, it is worth to mention the most popular ones: i.e. classical methods 


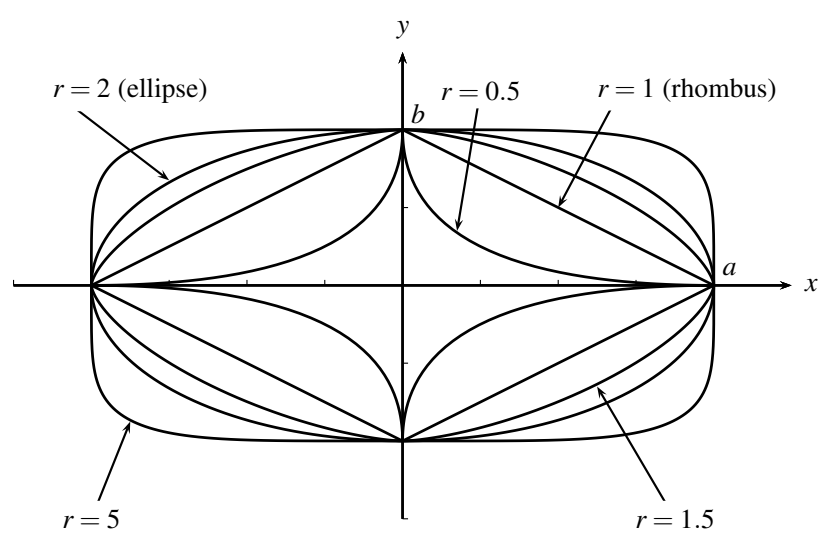

Fig. 3. Superellipses with semi-axes $a=4, b=2$ and different values of $r$.

[22], optimization for multi-band responses [23], iterative methods [24] for short-step transformers, and transformed variable methods using Richards' variable [25] and [26]. Of course, all of them have to deal with the problems of the involved impedance steps using full-wave electromagnetic (EM) simulations. All these responses can be easily translated to tapered waveguides using the technique shown in this paper, with the only change of using a different specification mask. The advantage is that a tapered line can achieve a similar performance as a multi-section transformer with a substantially shorter device. An additional advantage of the design method proposed in this work is that there is no need of performing any EM optimization, since there is always a circuital model accounting for the modal dispersion and the change of the waveguide shape.

Our approach tries to find the optimum performance using simpler methodology than the previous works. Moreover, the shortest possible length can be easily found according to specifications. Finally, easier fabrication is achieved because the proposed profile is a simple curve.

\section{TAPER Profile Using SuPERElLipses}

The idea of using superellipses is not new in engineering since it was used in architecture, typography and furniture design [27] among others. A superellipse is also known as a Lam curve after Gabriel Lam (well known for his general theory of curvilinear coordinates, and his notation and study of classes of ellipse-like curves). The superellipse is a general curve (see Fig. 3) with only one parameter $r$ ranging from $r \rightarrow 0$ (curve approaches the cartesian axes), and $r \rightarrow \infty$ (a rectangle). The general equation of a superellipse is

$$
\left(\frac{x}{a}\right)^{r}+\left(\frac{y}{b}\right)^{r}=1
$$

where $a$ and $b$ are the semi-axes of the superellipse, and $r$ is the parameter controlling the roundness of the ellipse. $r=1$ provides the rhombus (linear curve) and $r=2$ a true elliptic profile. Fig. 3 shows the shape of the superellipses for $a=2 b$ and several values of $r$.

However, the curvature controlled with the only parameter $r$ does not give enough freedom to generate a profile with competitive behavior. Further generalizations are used in

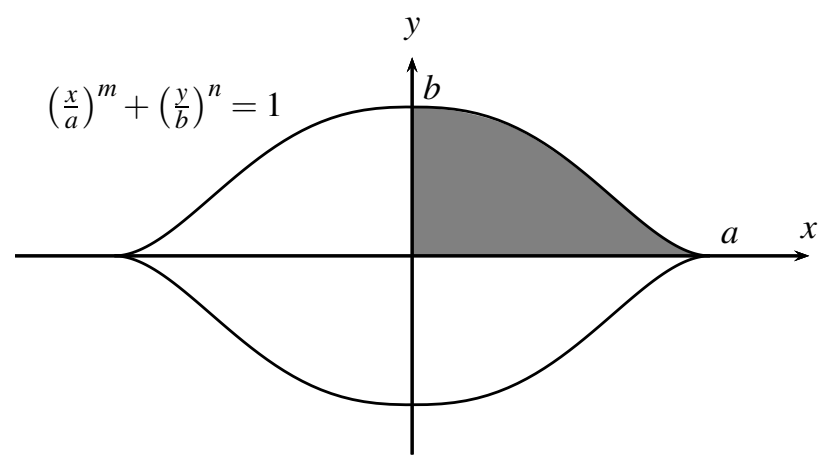

Fig. 4. Generalized superellipse with semi-axes $a=3$ and $b=1.5$ and exponents $m=2.5$ and $n=0.5$. The shadowed quadrant will be used for the taper profile.

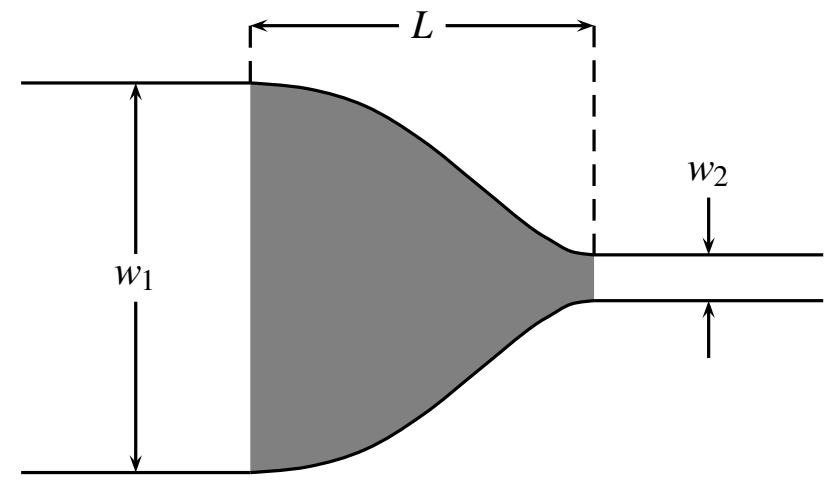

Fig. 5. Taper profile formed mirroring and offsetting the generalized superellipse quadrant of Fig. 4.

several fields like modeling in botany or metamaterials (see [28], [29] and [30]). The equation we are going to use for tapers is a quadrant of a generalized superellipse, whose expression is

$$
\left(\frac{x}{a}\right)^{m}+\left(\frac{y}{b}\right)^{n}=1
$$

where $m, n \in \mathbb{R}^{+}$are constants controlling the curvature of each dimension. The advantage of using the quadrant of a superellipse is twofold: matching the curve at both ends is automatic, and secondly the curve does not change the sign of its tangent (true tapers are monotonic). A typical example of generalized superelliptic profile is shown in Fig. 4 where $m=2.5$ and $n=0.5$. The shadowed quadrant will be the profile of the taper. Mirroring and offsetting this quadrant we obtain the required taper as shown in Fig. 5. Extreme values for $m$ (i.e. $m=0$ or $m \rightarrow \infty$ ) lead to a sharp transition in the input side of the taper. The same occurs with $n$ and the output of the taper. Additionally, values of $m$ greater than one produce an outwards-curved (convex) junction with the $y$ axis. Values of $m$ lesser than one produce an inwards-curved (concave) junction with the $y$-axis. The same applies to $n$ and the $x$-axis junction. Therefore, the generalized superellipse can deal with impedance matching at least as well as a linear taper (generalized superellipse with $m=n=1$ ).

Curves like generalized superellipses with real-valued exponents provide a better fitting features than polynomials. This is because polynomials have integer order by definition. 


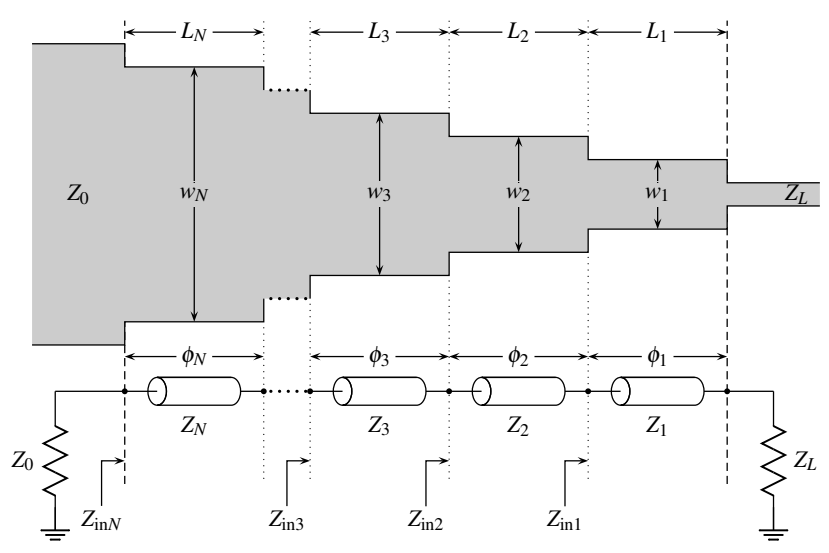

Fig. 6. Multi-section transformer and its equivalent transmission-line circuit representation.

Additionally, using polynomial approximations, it is quite difficult to avoid derivative changes that would produce a wiggly tapered line. The typical approach of dividing the curve in splines will increase the unknowns of the problem as the number of the splines increases. Therefore, the use of just two parameters for the whole profile provides a simple solution with excellent performance.

\section{Design Strategy for Planar Tapers}

Let us assume that the taper profile is given. The physical geometry of a taper is continuous, like the one shown in Fig. 5, but it can be studied as a discrete multi-section transformer where the number of sections $N$ tends to infinity (see Fig. 6). This structure can be simulated with available EM tools. However, the solver has to be harnessed to an optimizer to obtain the optimal parameters of the superelliptic profile. Many simulations need to be performed during the optimization and a fast equivalent circuit simulator is required. Therefore, a segmentation strategy is chosen, being quite accurate and fast enough for our purpose.

Let be $L_{i}=L / N$ the length of each section, where $L$ is the total length of the taper and $N$ the number of sections. For the sake of simplicity, the sections will have the same length $L_{1}=L_{2}=\cdots L_{N}$. The number of sections $N$ has to be high enough to neglect the steps between sections, namely, each $i$-th section has width $w_{i}$, and $N$ is chosen so that

$$
\left|w_{i}-w_{i+1}\right|<\epsilon \quad \forall i=1, \ldots, N-1
$$

where $\epsilon$ is the maximum allowed step that can be neglected in the analysis (e.g. $\epsilon<\lambda_{g} / 100$ being $\lambda_{g}$ is the guided wavelength at the minimum frequency of interest). This can be done because the effect of a change in width is very small for planar structures. The physical model can be analyzed using the electrical model shown at the bottom of Fig. 6 . This model is a cascade of $N$ sections of transmission lines, where each section has a characteristic impedance $Z_{i}$ given by the relationship between the width and the impedance for the considered substrate [7], [31]. Here, the dispersion can be taken into account since $\varepsilon_{\text {eff }}$ (and therefore $Z_{i}$ ) is not constant with frequency in applications with wide bandwidth. The procedure to obtain the reflection coefficient starts with

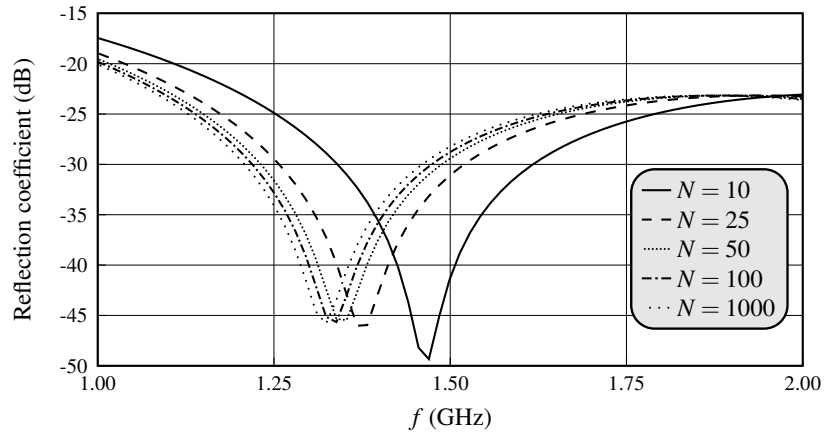

Fig. 7. Reflection coefficient computed varying the number of sections chosen to divide the geometrical shape of the taper. These curves correspond to the superelliptic example considered in subsection VI-A.

the computation of the input impedance of the first line $Z_{\text {in } 1}$ as

$$
Z_{\text {in } 1}=Z_{1} \frac{Z_{L}+j Z_{1} \tan \phi_{1}}{Z_{1}+j Z_{L} \tan \phi_{1}}
$$

and the result is stored for each frequency point. Needless to say that the electrical length $\phi_{1}=\beta_{1} L_{1}$ is computed assuming dispersion, that is included in the computation of $\beta_{1}$.

Now, $Z_{\text {in1 }}$ becomes the load impedance when $Z_{\text {in2 }}$ is computed and in general, at the step $i$-th the input impedance of section $i$ is computed as

$$
Z_{\text {in } i}=Z_{i} \frac{Z_{\text {in } i-1}+j Z_{i} \tan \phi_{i}}{Z_{i}+j Z_{\text {in } i-1} \tan \phi_{i}}
$$

and the process is repeated up to section $N$ where $Z_{\operatorname{in} N}$ is obtained.

Finally, the reflection coefficient can be computed as

$$
\Gamma_{\text {in }}=\frac{Z_{\text {in } N}-Z_{0}}{Z_{\text {in } N}+Z_{0}}
$$

A typical set of curves obtained to check the convergence of the proposed analysis method against the number of sections is shown in Fig. 7.

\section{Optimization Algorithm}

Once the number of steps $N$ has been properly chosen, the width for each section can be obtained using a superelliptic profile with parameters $m$ and $n$ using a simple function. The input parameters of the function are $\left(N, m, n, w_{N}, w_{1}\right)$. The length of the taper is $L=a$ ( $a$ being the semi-major axis of the generalized superellipse), and $b=\left(w_{N}-w_{1}\right) / 2$ ( $b$ being the semi-minor axis), as can be deduced from Fig. 5 and Fig. 6 . In order to avoid any step at the beginning or at the end of the taper, we assume that $w_{N}=w_{0}, w_{0}$ being the width of the line with $Z_{0}$ we are trying to match to the line with $Z_{L}$. For the same reason, the width of the first section $w_{1}=w_{L}$, where $w_{L}$ is the width of the line with characteristic impedance $Z_{L}$. Therefore, the taper starts with $w_{N}$ and smoothly changes its width to finally reach $w_{1}$. If we assume $w_{N}>w_{1}$ as it is drawn in Fig. 6, the function giving the width of $i$-th section is

$$
w_{i}=w_{1}+\left(w_{N}-w_{1}\right)\left[1-\left(\frac{i}{N}\right)^{m}\right]^{1 / n}
$$


where we have assumed all sections having the same length for the sake of simplicity.

Once the curve is chosen, two things are required. First, an EM simulator capable of dealing with such profile with a fair accuracy, and secondly an optimization algorithm providing a good convergence. The former is the main problem. However, for microstrip structures, a transmission-line approach can be used with intensive segmentation, and neglecting the small steps in the structure as explained in the previous section. If the number of segments is high, the simulated structure behaves as an smooth and continuous device. Therefore, the designer has to choose the maximum impedance step that can be neglected, and enforce a partition with such small steps. After a convergence study, the number of segments needed for accurate simulations can be safely fixed. A typical convergence study was shown in Fig. 7, where a number of steps higher than 100 (i.e. $N \geq 100$ ) does not produce a high impact on the response for this particular case.

The optimization part of the problem has a wide range of possibilities. Since the number of involved optimization parameters is small, the authors chose a non-linear leastsquares method

$$
\min _{m, n}\|\mathbf{E}\|_{2}^{2}=\min _{m, n} \sum_{i=1}^{N_{\text {freq }}} e_{i}^{2}
$$

where $\mathbf{E}$ is the error function with $N_{\text {freq }}$ components $e_{i}$ (the number of points in the frequency sweep), and each component is defined as

$$
e_{i}=\left\{\begin{array}{lr}
S_{11}\left(f_{i}\right)-M\left(f_{i}\right), & \text { if } S_{11}\left(f_{i}\right)-M\left(f_{i}\right)>0, \\
0 & \text { otherwise }
\end{array}\right.
$$

where $M\left(f_{i}\right)$ and $S_{11}\left(f_{i}\right)$ are the values of the mask and of the reflection coefficient (in logarithmic units) at the $i$-th frequency point, respectively. The reflection coefficient $S_{11}$ changes not only with frequency but with the shape of the taper, that ultimately depends on $m$ and $n$.

The algorithm used to solve the minimization problem is quasi-Newton, and the Jacobian matrix can be obtained both numerically or analytically (if a segmentation of the problem with uniform transmission lines is used). The auxiliary matrix needed as the Jacobian in this algorithm is the Jacobian of the reflection coefficient

$$
\mathbf{J}=\left(\begin{array}{ll}
\frac{\partial S_{11}}{\partial m} & \frac{\partial S_{11}}{\partial n}
\end{array}\right)
$$

The computation of $\mathbf{J}$ for each frequency point speeds up the optimization algorithm if it is computed efficiently. Obviously it will depend on the approach used for the analysis of the structure, but in any case it can be used as a good estimation if computed using approximate formulas. Applying wise approximations in the EM simulation, the computation of $S_{11}$ and its Jacobian can be performed at very high speed as follows.

Let be $\Gamma_{\text {in }}=S_{11}$ the reflection coefficient of the taper. If the goal is to obtain an approximation of the Jacobian with respect to $m$ and $n$, we first notice that there is a dependence of the width with $m$ and $n$, namely $w(m, n)$ given by (7) for each section. The next step is to obtain the electrical length $\phi$,

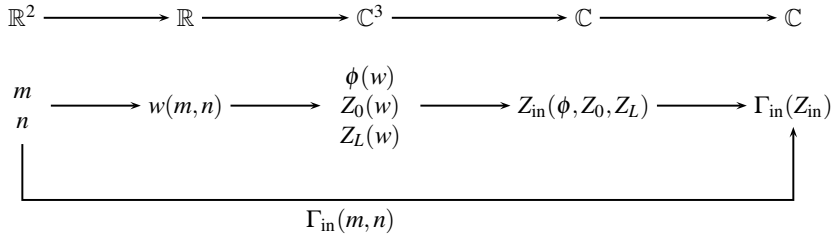

Fig. 8. Dependence among different variables used to apply the multivariate chain's rule to obtain the Jacobian matrix.

the characteristic impedance $Z_{0}$ and the load impedance $Z_{L}$ from $w$ using the classical formulas applied to each uniform $i$-th section [7], [31]; and recalling that the load impedance of the $i$-th section is the input inpedance of the $(i-1)$-th section as explained before. In our case, we used formulas accounting for finite thickness of the microstrip conductor and dispersion effects, with the aim of obtaining the highest accuracy.

It is clear that from the electrical length and the characteristic impedance of the $i$-th section $\phi_{i}, Z_{i}$ and the input impedance of the $(i-1)$-th section $Z_{\text {in } i-1}$, the input impedance $i$-th section $Z_{\text {in } i}$ can be obtained using (5) and $\Gamma_{\text {in } i}$ using (6). Therefore, the whole chain is established and its diagram is shown in Fig. 8. Applying the chain's rule and dropping the subindex $i$ to avoid cumbersome notation we have:

$$
\begin{aligned}
& \mathbf{J}=\left(\frac{\partial \Gamma_{\text {in }}}{\partial m} \frac{\partial \Gamma_{\text {in }}}{\partial n}\right)= \\
& \frac{\partial \Gamma_{\text {in }}}{\partial Z_{\text {in }}} \cdot\left(\frac{\partial Z_{\text {in }}}{\partial \phi} \frac{\partial Z_{\text {in }}}{\partial Z_{0}} \frac{\partial Z_{\text {in }}}{\partial Z_{L}}\right) \cdot\left(\begin{array}{c}
\frac{\partial \phi}{\partial w} \\
\frac{\partial Z_{0}}{\partial w} \\
\frac{\partial Z_{L}}{\partial w}
\end{array}\right) \cdot\left(\frac{\partial w}{\partial m} \frac{\partial w}{\partial n}\right)
\end{aligned}
$$

where each term can be computed analytically for each section and for each frequency point.

Now, we are ready to write the direction $\mathbf{v}$ of the maximum variation of the error function [32] to seek the extremum using (8) and (9):

$$
\mathbf{v}=\nabla\|\mathbf{E}\|_{2}^{2}=\left(\frac{\partial\|\mathbf{E}\|_{2}^{2}}{\partial m} \frac{\partial\|\mathbf{E}\|_{2}^{2}}{\partial n}\right)=2 \sum_{i=1}^{N_{\text {freq }}} \mathbf{J}_{i} e_{i}
$$

Namely, the direction of maximum variation is proportional to $\mathbf{J}_{i}$ (Jacobian at $i$-th frequency point) given by (11) as expected. It is important to notice that all functions involved (e.g. $Z_{\text {in }}, \phi$, etc.) have to be computed for each section and for each frequency point. Therefore, if the functions are stored, the memory needed for each function is $N_{\text {freq }} \times N$ complex numbers in general.

\section{Strategy for WaVeguide TApers}

Usually, an adaptation between two standard rectangular waveguides is needed for certain applications. Here, we cannot apply the typical planar approaches, since the waveguide geometry depends on the two dimensions (width and height) of the waveguide. There are several typical definitions of the characteristic impedance (power-voltage, power-current and voltage-current), all of them depending on $a$ and $b$ and the modal impedance [20]. However, using the optimization 
technique for planar tapers to both dimensions separately, waveguide tapers can be designed without further difficulty choosing any of the aforementioned waveguide impedance definitions.

The optimization problem is formally the same as the one described for planar tapers, with the only difference that there are two variables $(m, n)$ for each rectangular waveguide dimension (four in total). This sole difference does not mean a great problem, since a 4-variable optimization problem can be handled comfortably using the same optimization technique used for planar tapers. In order to avoid complex analytical expressions for the Jacobian matrix, in this case the computation has been carried out numerically.

The main problem arises in the analysis part of the proposed waveguide taper. Here, we cannot neglect the steps when the taper is divided in a multi-section structure, each one having a different rectangular cross section. The solution is to take into account such transitions involving a change in terms of height and width. Applying the superposition principle, we are going to assume that a waveguide step in height and width can be decomposed into two simpler steps (one in width and one in height). Since the partition provides small steps, we can use the asymptotic expressions given in [8]. Let be a step from a waveguide with dimensions $a$ (width) and $b$ (height), guided wavelength $\lambda_{g}$ and characteristic impedance $Z_{0}$ to a waveguide with parameters $a^{\prime}, b^{\prime}, \lambda_{g}^{\prime}$ and $Z_{0}^{\prime}$. The change in width leads to a change in the impedance following the expression

$$
\frac{Z_{0}^{\prime}}{Z_{0}} \approx \frac{\lambda_{g}^{\prime} a^{\prime}}{\lambda_{g} a}\left(1+\gamma+\frac{\gamma^{2}}{2}\right) \quad \forall \gamma \ll 1
$$

where

$$
\gamma=1-\frac{a^{\prime}}{a}
$$

The step itself can be modeled as a parallel reactance $Z_{\text {step }}=$ $j X$ whose asymptotic expression for $\gamma \ll 1$ is

$$
\frac{Z_{0}}{X} \approx \frac{\lambda_{g}}{2 a} \frac{\gamma^{2}(1+\gamma) \ln \frac{2}{\gamma}}{1-\frac{\gamma}{2}}\left(1-\frac{27}{8} \frac{Q+Q^{\prime}}{1+8 \ln \frac{2}{\gamma}}\right)
$$

where

$$
Q=1-\sqrt{1-\left(\frac{2 a}{3 \lambda}\right)^{2}} \quad Q^{\prime}=1-\sqrt{1-\left(\frac{2 a^{\prime}}{3 \lambda}\right)^{2}}
$$

and $\lambda$ is the free-space wavelength. The step in height changes the characteristic admittance as

$$
\frac{Y_{0}}{Y_{0}^{\prime}}=\frac{b^{\prime}}{b}=1-\delta
$$

and the step itself can be modeled as a parallel susceptance $Y_{\text {step }}=j B$, whose asymptotic expression for $\delta \ll 1$ (small step) is

$$
\frac{B}{Y_{0}} \approx \frac{2 b}{\lambda_{g}}\left(\frac{\delta}{2}\right)^{2}\left[\frac{2 \ln \frac{2}{\delta}}{1-\delta}+1+\frac{17}{16}\left(\frac{b}{\lambda_{g}}\right)^{2}\right]
$$

The formulas are really accurate provided the step is small [8], and the model is extremely fast because the computation effort of the previous formulas for each step is negligible. The model is shown in Fig. 9, where the only difference with the planar

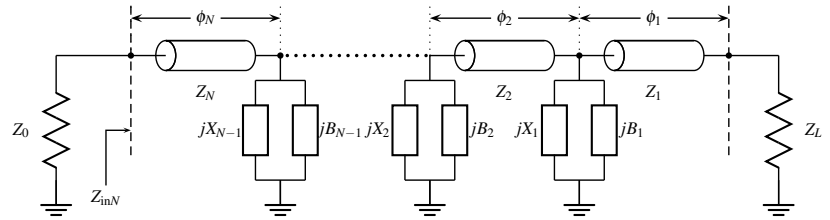

Fig. 9. Equivalent transmission-line circuit representation of a multisection transformer

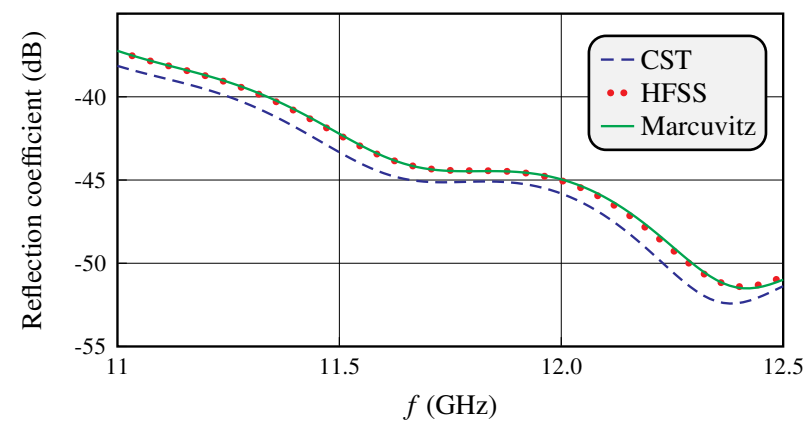

Fig. 10. Linear waveguide taper WR-90 to WR-62. The blue dashed line is the EM simulation using CST. The dotted red line is the EM simulation using HFSS. The solid green line shows the simulation using Marcuvitz formulas.

model are the shunt reactances (modeling the corresponding waveguide steps or transitions).

In order to check the simulator built with Marcuvitz's formulas against commercial simulators, a linear taper between WR-90 and WR-62 standard waveguides has been used as a benchmark. The taper length chosen for this example is $150 \mathrm{~mm}$, which is the typical length of a commercial taper. Fig. 10 shows the simulation of the linear taper using HFSS and CST compared with the results given by Marcuvitz's formulas. The results agree very well taking into account that Marcuvitz's formulas provide the results in less than $1 \mathrm{~s}$ (with a standard dual-core PC at $2.2 \mathrm{GHz}$ with $4 \mathrm{~GB}$ of RAM), even though a partition with 400 sections has been used. It should be noted that the way the commercial EM simulators discretize the volume leads to different solutions, depending on whether the discretization follows the true shape or not. Therefore, instead using mesh sizes, a convergence criteria based on the solution stability is normally used.

\section{DESIGN EXAMPLES}

\section{A. Planar Taper}

In order to check the performance of the novel impedance matching structure, a taper based on a generalized superellipse is designed to provide a return loss level $R L>25 \mathrm{~dB}$ in the range from 1 to $3 \mathrm{GHz}$ between two impedances of $Z_{0}=50$ $\Omega$ and $Z_{L}=100 \Omega$. For this example a RO4003 substrate is used. The minimum matching provides the minimum value 


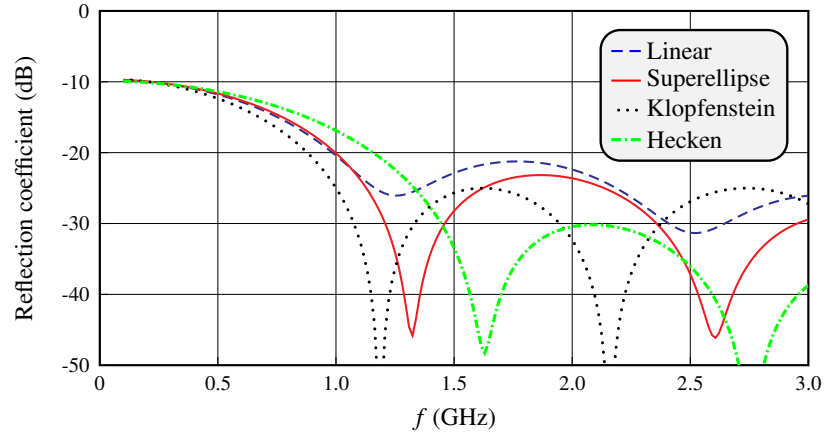

Fig. 11. Reflection response of the linear taper compared with the superelliptical solution. The dotted line shows the analytic equirriple solution (Klopfenstein) and the dash-dotted line shows the simulation of the nearoptimum solution given in [10].

for the length $L$ of the taper [4]:

$$
\begin{aligned}
\Gamma_{m} & =10^{-R L / 20} \\
\Gamma_{0} & =\frac{Z_{L}-Z_{0}}{Z_{L}+Z_{0}} \approx \frac{1}{2} \ln \frac{Z_{L}}{Z_{0}} \\
A & =\cosh ^{-1}\left(\Gamma_{0} / \Gamma_{m}\right) \\
L & =\frac{A c_{0}}{2 \pi f_{\min } \sqrt{\varepsilon_{r_{\text {eff }}}}}
\end{aligned}
$$

where $\Gamma_{m}$ is the maximum reflection coefficient allowed in the equirriple design, $c_{0}$ is the speed of light in the vacuum and $f_{\min }$ is the minimum frequency at which the specification $\Gamma_{m}$ is met. These equations provide a length of $L=72.6$ $\mathrm{mm}$. However, it is expected that the Klopfenstein taper will not provide $\left|S_{11}\right|<-25 \mathrm{~dB}$ in the EM simulation, because of all the assumptions in the related design method. The first one is the non-dispersive media assumed in the calculations, the second one is the approximations of the equation (Ricatti's equation) of the reflection coefficient (see [2], [4]), and the third one is that the solution is given in terms of an impedance profile (24) which is synthesized at a given frequency (not broadband).

The design method proposed in this work requires a mask (we used a constant mask to obtain a solution close to Klopfenstein's), and an initial guess of the variables to be optimized (we used $m=n=1$ giving the linear profile as the starting point).

Optimizing the superelliptical profile, the method will provide (in less than 1 second) the optimal parameters: $m=$ 0.94084 and $n=0.76022$. Fig. 11 shows the difference in performance of the linear, the superelliptical and the nearoptimum tapers with the ideal Klopfenstein's response given by the analytic formula

$$
\Gamma(\beta L)=\Gamma_{0} \frac{\cos \sqrt{(\beta L)^{2}-A^{2}}}{\cosh A}
$$

As expected, the superelliptical profile provides an improvement over the linear taper, which is the starting point of the optimization we have performed. In this example, the improvement is obtained in the whole frequency range. The limit for the performance is the equirriple solution given by the Klopfenstein's taper. However, (23) is analytical and does not

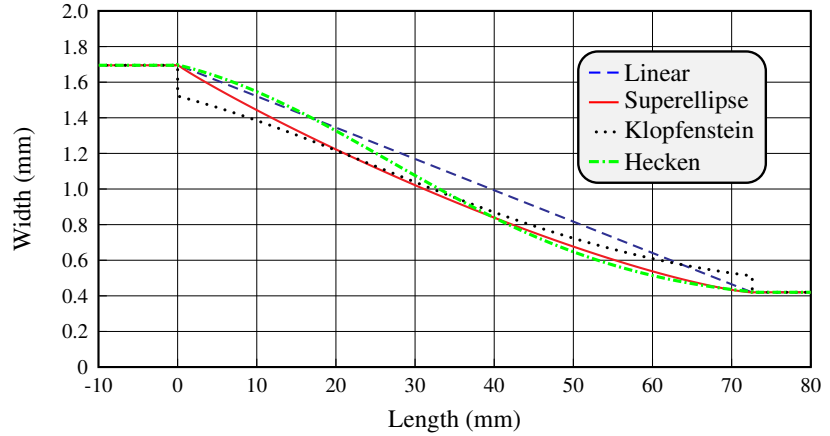

Fig. 12. Comparison between the linear profile and the optimized solution The dotted line shows the analytic solution showing the impedance steps. The dash-dotted line shows the near-optimum profile. Only one side of the taper is plotted. The other side is symmetric with respect to the horizontal axis.

consider more realistic issues. The superelliptic solution has been obtained after optimization using a real profile segmented in 2000 sections. In each section, the effective dielectric constant and the phase constant have been computed using empirical formulas [7], [31], assuming their true dependence with the frequency variation to better approximate the final response.

The profile of the Klopfenstein's taper can be computed using the well-known formulas [3]

$\ln Z(z)=\left\{\begin{array}{l}\ln Z_{0}, \quad z \leq 0 \\ \frac{1}{2} \ln \left(Z_{0} Z_{L}\right)+\frac{A^{2} \Gamma_{0}}{\cosh A} \phi\left(\frac{2 z}{L}-1, A\right), \quad 0<z<L \\ \ln Z_{L}, \quad z \geq L\end{array}\right.$

where

$\phi(x, A)=-\phi(-x, A)=\int_{0}^{x} \frac{I_{1}\left(A \sqrt{1-y^{2}}\right)}{A \sqrt{1-y^{2}}} d y \quad \forall|x|<1$

where $I_{1}(x)$ is the modified Bessel function. $\phi(x, A)$ can be computed very rapidly, since there is an efficient algorithm detailed in [33].

The profile of the linear taper (initial point of the optimization) is compared versus the superelliptic taper in Fig. 12. In the same figure, the Klopfenstein's solution shows the steps inherent to the equirriple solution. As a curiosity, Hecken's solution appears in the same plot showing strong similarity with the superelliptic profile in the second half of the taper. In the first half of the taper, the superellipse rapidly approaches the Klopfenstein profile.

It is worth to stress that the optimization algorithm seeks an $m$ and $n$ values in each iteration, then the profile is simulated and the error $\mathbf{E}$ referred to the mask is computed. In the following iteration, the new $m$ and $n$ values will be sought in order to decrease the error function. Therefore, a fast simulator is strongly recommended. The segmentation technique already explained before (see section III) gives a good approximation, and a transmission-line simulator has been implemented for this purpose.

The code for the analysis of the taper has been written in the MATLAB ${ }^{\mathrm{TM}}$ environment. For the optimization process, 


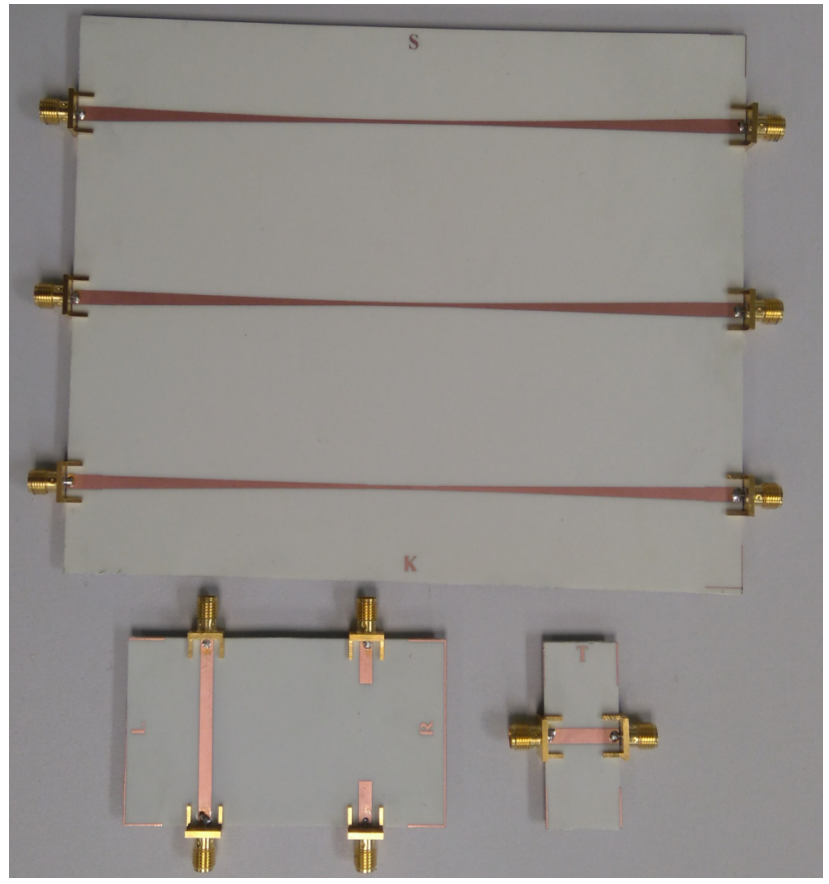

Fig. 13. Manufactured prototypes (circuit board at the top) and the TRL calibration kit (circuits at the bottom). The upper circuit in the board is the superelliptic taper (near the letter S), the middle one is the linear taper and the lower circuit is the Klopfenstein taper (near the letter $\mathrm{K}$ ).

the non-linear least-squares algorithm has been used. Upper and lower bounds for the values of $m, n$ are set to practical values (lower bound 0.1 and upper bound 10 will suffice). For the initial values in the optimization, as explained, $m=n=1$ (linear taper) is used as the starting point.

Each taper (linear, superelliptic and Klopfenstein) simulated in Fig. 11 has been manufactured in a cascaded backto-back configuration (see Fig. 13, where the employed TRL calibration kit is also shown). Using this set-up, the measurement can be carried out with a $50 \Omega$ calibrated VNA. This method allows to fairly compare the performances of the three tapers using the same calibration kit. Ideally, the performance of a back-to-back configuration provides a $3 \mathrm{~dB}$ higher reflection level. The measured reflection of manufactured prototypes is shown in Fig. 14. The results show how the superelliptic taper is clearly superior with respect to Klopfenstein's at almost all frequencies. The linear taper is also worse below $2.1 \mathrm{GHz}$. It is important to note that the impedance steps in the Klopfenstein taper has a negative impact in its performance (at some frequencies, even the linear taper is better).

\section{B. Waveguide Taper}

The proposed solution has been validated with two manufactured prototypes in waveguide technology. Both are WR-90 to WR-62 waveguide tapers with length $L=50 \mathrm{~mm}$. One is manufactured as a single piece using the wire electrical discharge machining (EDM) technique. The other one has been manufactured in two equal pieces using milling (see Fig. 15). The final goal of the design is to find the best possible solution

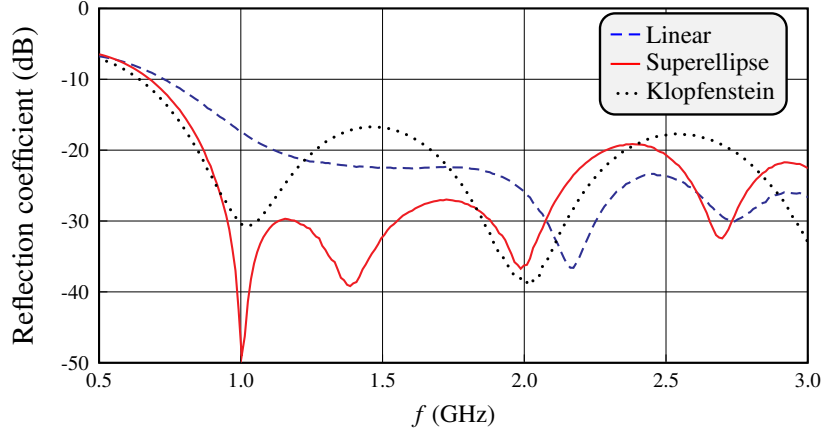

Fig. 14. Measured reflection response of the three tapers built in a back-toback configuration.

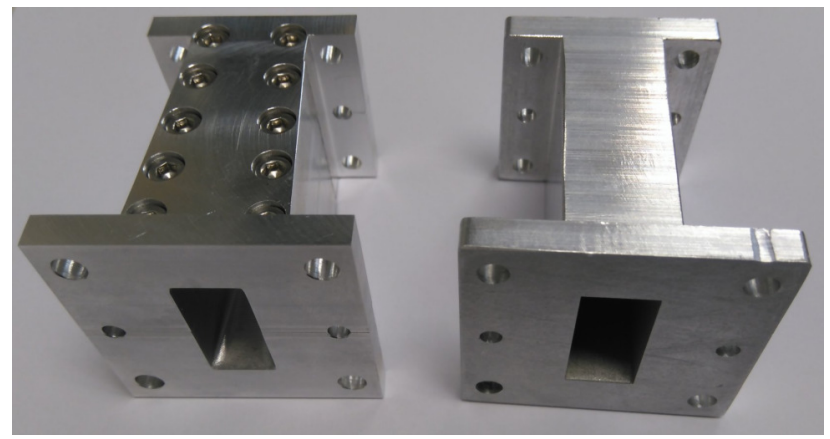

Fig. 15. Manufactured tapers in two pieces using milling (left) and in one single piece using wire EDM (right). Both are $50 \mathrm{~mm}$ long tapers matching WR-90 to WR-62 standard waveguides.

with $S_{11}<-40 \mathrm{~dB}$ in the band $11-12.5 \mathrm{GHz}$, which is common to both standard waveguides. With these goals, the solution will improve the response of the commercial $150 \mathrm{~mm}$ taper considered in Fig. 10. The optimization procedure gives as the optimal point the following values

$$
\begin{array}{ll}
m_{1}=0.6587 & n_{1}=0.5259 \\
m_{2}=0.7132 & n_{2}=0.6845
\end{array}
$$

where $m_{1}$ and $n_{1}$ are the parameters of the superellipse matching the waveguide widths, and $m_{2}, n_{2}$ are the parameters related with the height. The response of the taper using the three simulators (two EM full-wave commercial tools and one circuit simulator using Marcuvitz's formulas) is shown in Fig. 16. All simulations reached $S_{11}<-40 \mathrm{~dB}$. However, the observed differences rely on the 3D-meshing of the commercial simulators, that do not exactly follow the profile due to the employed discretization methods, and to the fact that the circuit simulator uses the approximations outlined in section V. Nevertheless, it is important to stress that the observed difference is small in linear units, since the low level of reflection is quite extreme.

Once the two prototypes were built (as shown in Fig. 15), they have been measured. However, the measurement technique is not unique. If the taper is connected to the vector network analyzer (VNA) with the WR-90 port, the load has to be connected to the WR-62 side of the taper. Conversely, if the VNA is connected to the WR-62 port, the load has to 


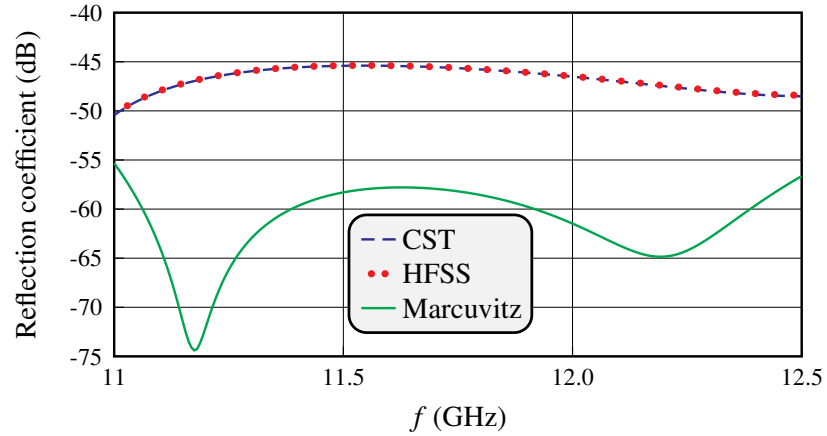

Fig. 16. Three simulations of the optimal waveguide taper with $L=5 \mathrm{~mm}$. CST and HFSS give the same results with 3D meshing. The circuit simulator using Marcuvitz's formulas used 400 cascaded lines with the corresponding steps.

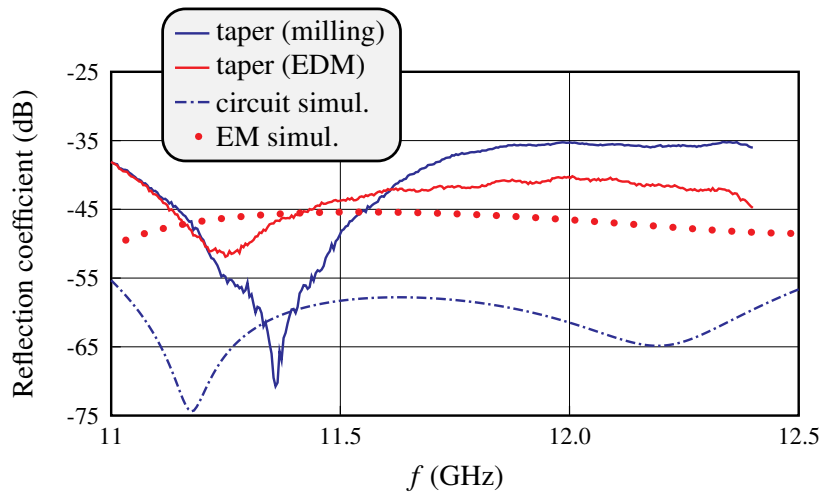

Fig. 17. Measurements of the reflection coefficient of the waveguide tapers from the WR-90 port. A WR-62 matching load has been used. EM and circuit simulations are also shown.

be connected to the WR-90 side of the taper. The loads are different and the response differs depending on the used loads.

The results in Fig. 17 show a good performance of both tapers, whose reflection has been measured from the WR-90 port using a WR-62 matching load at the other port of the tapers. It also shows how the predictions of the simulators are quite different. The circuit simulator predicts the reflection zero, whereas the EM simulation approaches the average level.

The difference between the measured tapers and the simulations are due to two main sources: the frequency response of the matching load connected to the taper (it has a response near the level of our goal for the reflection coefficient) and the mechanization imperfections.

In order to see the out-of-band performance, measurements of the tapers from the WR-62 port having loaded the WR-90 ports are shown in Fig. 18. The calibration kit used is different as well as the frequency range. Therefore, the differences with respect to Fig. 17 are easily justifiable. The reflection level is clearly maintained.

There are alternative measurement techniques for tapers, as it has been shown in the microstrip example. If two equal tapers are available, they can be connected in a back-toback configuration and we can remove the load from the measurements. However, there are two possible configurations:

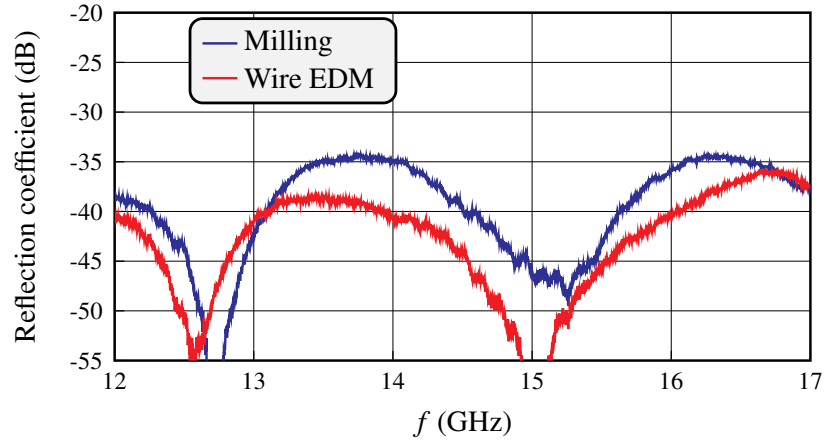

Fig. 18. Measurements of the reflection coefficient of the waveguide tapers from the WR-62 port. A WR-90 matching load has been used.

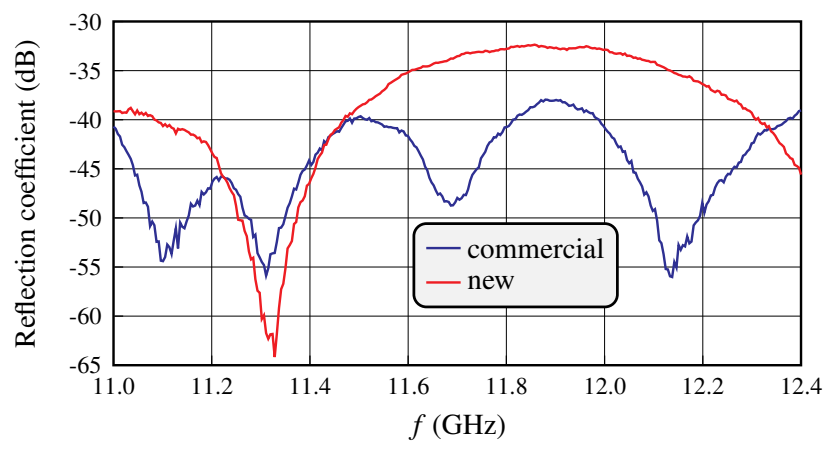

Fig. 19. Back-to-back configuration joining the tapers from the WR-62 ports. Commercial tapers with length $L=150 \mathrm{~mm}$ are also measured for comparison purposes.

joining the tapers from the WR-90 ports or from the WR- 62 ones. The frequency range will be different and the results may also vary. We have two tapers with the only difference of the fabrication technique. Moreover, two equal commercial tapers with the same ports as our prototypes, but with $L=150$ $\mathrm{mm}$ each one, are also available. For these measurements in back-to-back configuration we used a two-port calibration and measurement setup, thus removing the matching load and its influence from the measurements themselves.

Figs. 19 and 20 show a comparison of experimental results for the back-to-back configurations with the commercial tapers, which are three times as long as the designed tapers

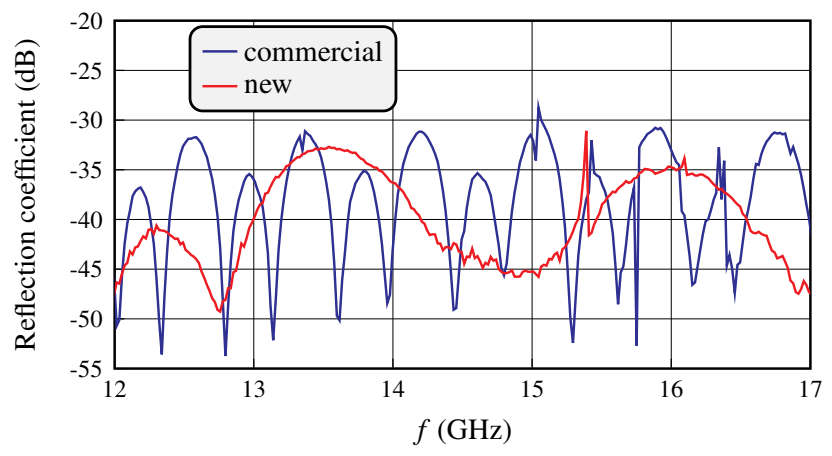

Fig. 20. Back-to-back configuration joining the tapers from the WR-90 ports. Commercial tapers with length $L=150 \mathrm{~mm}$ are also measured for comparison purposes. 


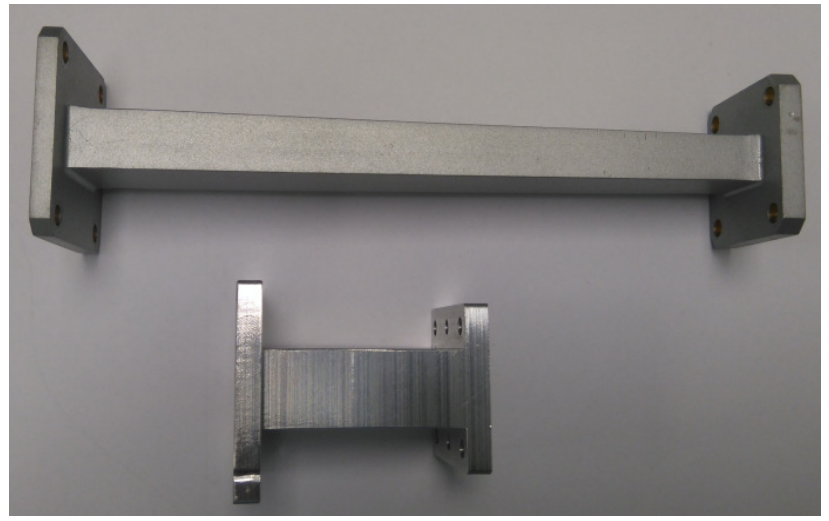

Fig. 21. Commercial taper (top) compared with one of the manufactured prototypes (bottom).

(see both sizes in Fig. 21). The reflection level is similar despite the difference in length.

\section{CONCLUSION}

A novel method to design tapered lines based on superellipses has been proposed. In microstrip technology, the superelliptic taper has a superior performance in most of the designed bandwidth, once compared with the equirriple optimal solution and the traditionally used linear taper. The enhanced performance and the related short design time justifies the method proposed in this paper. The measured responses show the importance of smooth profiles with optimized behavior in tapered lines. For waveguide tapers, two prototypes based on the generalized superellipse geometry have been also manufactured. Their performance is similar when compared with commercial tapers three times longer.

\section{REFERENCES}

[1] G. L. Matthaei, L. Young, and E. M. T. Jones, Microwave Filters, Impedance Matching Networks, and Coupling Structures. New Jersey: Artech House, 1980.

[2] R. E. Collin, Foundation for Microwave Engineering, 2nd ed. New Jersey: Wiley-IEEE Press, 2000.

[3] D. M. Pozar, Microwave Engineering, 3rd ed. New Jersey: John Wiley \& Sons, 2005.

[4] R. W. Klopfenstein, "A transmission line taper of improved design," Proceedings of the IRE, vol. 44, no. 1, pp. 31-35, Jan 1956.

[5] R. E. Collin, "The optimum tapered transmission line matching section," Proceedings of the IRE, vol. 44, no. 4, pp. 539-548, April 1956.

[6] D. Kajfez and J. O. Prewitt, "Correction to "a transmission line taper of improved design" (letters)," IEEE Trans. Microw. Theory Techn., vol. 21, no. 5, pp. 364-364, May 1973.

[7] K. C. Gupta, R. Garg, I. Bahl, and P. Bhartia, Microstrip Lines and Slotlines, 2nd ed. Norwood, MA: Artech House, 1996.

[8] N. Marcuvitz, Waveguide Handbook. Stevenage, UK: Peter Peregrinus Ltd., 1986.

[9] L. Solymar, "A note on the optimum design of non-uniform transmission lines," Proceedings of the IEE - Part C: Monographs, vol. 107, no. 11, pp. 100-104, March 1960.

[10] R. P. Hecken, "A near-optimum matching section without discontinuities," IEEE Trans. Microw. Theory Techn., vol. 20, no. 11, pp. 734-739, Nov 1972.

[11] J. P. Mahon and R. S. Elliott, "Tapered transmission lines with a controlled ripple response," IEEE Trans. Microw. Theory Techn., vol. 38, no. 10, pp. 1415-1420, Oct 1990.

[12] L. D. Landau, E. M. Lifshitz, and L. Pitaevskii, Electrodynamics of Continuous Media, 2nd ed., ser. Course of Theoretical Physics. Elsevier, 1984, vol. 8.
[13] R. Marques, F. Martin, and M. Sorolla, Metamaterials with Negative Parameters, ser. Microwave and Optical Engineering. Wiley Interscience, 2008.

[14] F. Martin, Artificial Transmission Lines for RF and Microwave Applications, ser. Microwave and Optical Engineering. Wiley, 2015.

[15] E. Hammerstad and O. Jensen, "Accurate models for microstrip computer-aided design," in IEEE MTT-S Int. Microwave Symp. Dig., May 1980, pp. 407-409.

[16] M. Kobayashi, "A dispersion formula satisfying recent requirements in microstrip CAD," IEEE Trans. Microw. Theory Techn., vol. 36, no. 8, pp. 1246-1250, Aug 1988.

[17] E. J. Park, "An efficient synthesis technique of tapered transmission line with loss and dispersion," IEEE Trans. Microw. Theory Techn., vol. 44, no. 3, pp. 462-465, Mar 1996.

[18] P. Pramanick and P. Bhartia, "Analysis and synthesis of tapered finlines," in IEEE MTT-S Int. Microwave Symp. Dig., May 1984, pp. 336338.

[19] A. Mantzke, S. Sdekum, and M. Leone, "Broadband equivalent-circuit model for non-uniform transmission lines," in 2015 IEEE International Symposium on Electromagnetic Compatibility (EMC), Aug 2015, pp. 600-605.

[20] J. Helszajn, Ridge Waveguides and Microwave Passive Components, ser. IEE Electromagnetic Waves. Stevenage, UK: The Institution of Engineering and Technology, 2000, vol. 49.

[21] S. Cogollos, J. Vague, V. E. Boria, and J. D. Martinez, "New design method of impedance matching networks based on tapered lines using generalized superellipses," in IEEE MTT-S Int. Microwave Symp. Dig., June 2017, pp. 1-4.

[22] S. Cohn, "Optimum design of stepped transmission-line transformers," IRE Trans. Microw. Theory and Techn., vol. 3, no. 3, pp. 16-20, Apr. 1955.

[23] U. Rosenberg, J. Bornemann, and S. Amari, "Design of dual-band waveguide transformers," in IEEE MTT-S Int. Microwave Symp. Dig., 2005, pp. 1215-1218.

[24] R. J. Cameron, C. M. Kudsia, and R. R. Mansour, Microwave Filters for Communication Systems: Fundamentals, Design and Applications. New Jersey: John Wiley \& Sons, 2007.

[25] H. Riblet, "General synthesis of quarter-wave impedance transformers," IRE Trans. Microw. Theory and Techn., vol. 5, no. 1, pp. 36-43, Jan. 1957.

[26] S. Cogollos, V. E. Boria, and J. D. Martinez, "Generalized short step transformers for multi-band impedance matching," in 2012 42nd European Microwave Conference, Oct 2012, pp. 380-383.

[27] M. Gardner, Mathematical Carnival. New York: Vintage Press, 1977.

[28] J. Gielis, "A generic geometric transformation that unifies a wide range of natural and abstract shapes," American Journal of Botany, vol. 90, no. 3, pp. 333-338, 2003.

[29] J. Gielis, B. Beirinckx, and E. Bastiaens, "Superquadrics with rational and irrational symmetry," in Proceedings of the Eighth ACM Symposium on Solid Modeling and Applications, ser. SM '03. New York, NY, USA: ACM, 2003, pp. 262-265. [Online]. Available: http://doi.acm.org/10.1145/781606.781647

[30] K. Yao, C. Li, and F. Li, "Design of electromagnetic cloaks for generalized superellipse using coordinate transformations," in 2008 International Workshop on Metamaterials, Nov 2008, pp. 122-125.

[31] B. C. Wadell, Transmission Line Design Handbook. Norwood, MA: Artech House, 1991.

[32] J. A. Snyman, Practical Mathematical Optimization: An Introduction to Basic Optimization Theory and Classical and New Gradient-Based Algorithms, ser. Applied Optimization. New York: Springer, 2005, vol. 97.

[33] M. A. Grossberg, "Extremely rapid computation of the Klopfenstein impedance taper," Proceedings of the IEEE, vol. 56, no. 9, pp. 16291630, Sept 1968. 


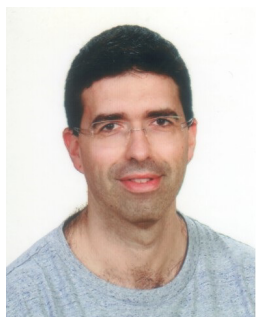

Santiago Cogollos was born in Valencia, Spain, on January 15, 1972. He received the degree in telecommunication engineering and the $\mathrm{Ph}$. D. degree from the Universitat Politècnica de València (UPV), Valencia, Spain, in 1996 and 2002, respectively. In 2000 he joined the Communications Department of the Universitat Politècnica de València, where he was an Assistant Lecturer from 2000 to 2001, a Lecturer from 2001 to 2002, and became an Associate Professor in 2002. He has collaborated with the European Space Research and Technology Centre of the European Space Agency in the development of modal analysis tools for payload systems in satellites. In 2005 he held a post doctoral research position working in the area of new synthesis techniques in filter design at University of Waterloo, Waterloo, Ont., Canada. His current research interests include applied electromagnetics, mathematical methods for electromagnetic theory, analytical and numerical methods for the analysis of microwave structures, and design of waveguide components for space applications

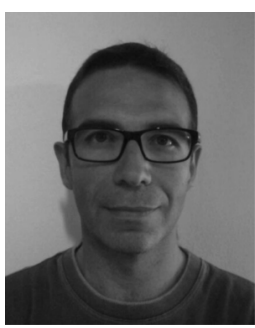

Joaquin Vague was born in Valencia, Spain, in 1979. He received the Electronic Engineering degree from the Universitat Politècnica de València (UPV), Valencia, Spain, in 2003. He is currently pursuing his $\mathrm{Ph}$. D. degree in Electronic Engineering. His main research interests are the design of microwave passive devices, in particular filters and nonreciprocal devices, as well as the improvement in manufacturing processes of RF and microwave components. He is currently a Technical Researcher in charge of several laboratories with the Department

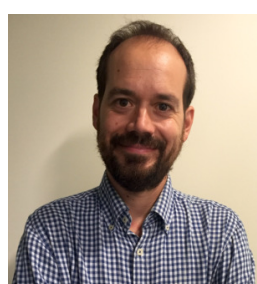

Jorge D. Martínez (M'09) was born in Murcia, Spain, on February 15, 1979, and he is Member of the IEEE, $\mathrm{PhD}$, and Associate Professor at the School of Telecommunication Engineering of the Polytechnic University of Valencia (UPV) since 2012. Previously, he received his degree in Telecommunication Engineering and the $\mathrm{PhD}$ degree from the same institution (UPV), in 2002 and 2008, respectively. He joined the Department of Electronic Engineering of the UPV in 2002 as a research fellow, and he became Assistant Professor in 2009. During 2007 he was a Research Visitor at XLIM, CNRS and University of Limoges (France), where he worked on the design and fabrication of RF MEMS components under the advice of Prof. Pierre Blondy. He is a researcher of the I3M R\&D institute at UPV, where he actively collaborates with the Microwave Applications Group (GAM). At I3M premises, he is now the Technical Responsible of the Laboratory for High Frequency Circuits Fabrication (LCAF) of the UPV, focused on Low Temperature Co-fired Ceramics (LTCC) and other related multi-layered technologies. His current research interests are focused on emerging technologies for reconfigurable microwave components with emphasis on tuneable filters and RF MEMS, and the design and fabrication of advanced microwave filters in planar and substrate integrated waveguide technologies, as well as the application of multi-layer fabrication technologies to $\mathrm{RF} /$ microwave and millimetre-wave applications

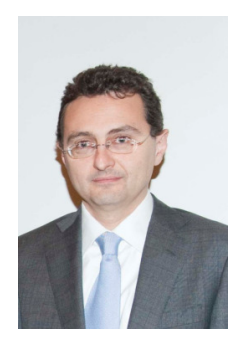

Vicente E. Boria (S'91-A'99-SM'02) was born in Valencia, Spain, on May 18, 1970. He received his "Ingeniero de Telecomunicación" degree (with first-class honors) and the "Doctor Ingeniero de Telecomunicación" degree from the Universidad Politécnica de Valencia, Valencia, Spain, in 1993 and 1997, respectively. In 1993 he joined the "Departamento de Comunicaciones", Universidad Politécnica de Valencia, where he has been Full Professor since 2003. In 1995 and 1996, he was holding a Spanish Trainee position with the European Space Research and Technology Centre, European Space Agency (ESTEC-ESA), Noordwijk, The Netherlands, where he was involved in the area of EM analysis and design of passive waveguide devices. He has authored or co-authored 10 chapters in technical textbooks, 160 papers in refereed international technical journals, and over 200 papers in international conference proceedings. His current research interests are focused on the analysis and automated design of passive components, left-handed and periodic structures, as well as on the simulation and measurement of high power effects in passive waveguide systems. Dr. Boria has been a member of the IEEE Microwave Theory and Techniques Society (IEEE MTT-S) and the IEEE Antennas and Propagation Society (IEEE AP-S) since 1992. He is member of the Editorial Boards of the IEEE Transactions on Microwave Theory and Techniques, IEEE Microwave and Wireless Components Letters, Proceedings of the IET (Microwaves, Antennas and Propagation), IET Electronics Letters and Radio Science. Presently, he serves as Associate Editor of IEEE Microwave and Wireless Components Letters and IET Electronics Letters. He is also a member of the Technical Committees of the IEEEMTT International Microwave Symposium and of the European Microwave Conference. 